\title{
UN MODELO COGNITIVO PARA LA CLASIFICACIÓN DE LOS COMPLEMENTOS DIRECTOS AFECTADOS EN ESPAÑOL
}

\begin{abstract}
Cuartero Otal Juan, Un modelo cognitivo para la clasificación de los complementos directos afectados en español [A Cognitive Model for the Classification of Affected Objects in Spanish]. Studia Romanica Posnaniensia, Adam Mickiewicz, University Press, Poznań, vol. XXXI: 2004, pp. 197-204. ISBN 83-232-1353-4, ISSN 0137-2475.

This article tries to justify an extended typology of the category 'affected object', analyzed by applying prototypical features and compared to other well-known versions (Anderson, 1977; Roberts, 1987 or Demonte, 1991). These data allow us to reflect about some combinatorial characteristics of Spanish verbs containing affected arguments in their argument structures, e. g. telicity of constructions (Tenny, 1987) or affectedness constraints (Jaeggli, 1986).
\end{abstract}

\section{INTRODUCCIÓN}

Los objetos afectados se incluyen propiamente entre las llamadas covert categories (Fillmore, 1968: 4), aquellas que no tienen realizaciones 'morfémicas' obvias pero sí una existencia objetiva que se manifiesta en restricciones de selección y posibilidades de transformación de los elementos. Hasta ahora se ha dedicado atención al tema desde muchos puntos de vista que incluyen la delimitación de casos semánticos, estudios sobre la transitividad y con especial interés a los fenómenos de selección y de transformación (Anderson, 1977; Jaeggli, 1986; Tenny, 1987; Zubizarreta, 1987; Fellbaum y Zribi-Hertz, 1989; Doron y Rappaport-Hovav, 1991; Demonte, 1991; Campos, 1999 etc.). En ellos se encuentran diversos acercamientos al concepto objeto afectado, en unos casos como descripciones por intensión y en otros casos por extensión. Si bien se observa claramente que los resultados de los trabajos avanzan basándose en el conocimiento de los previos, también se nota al comparar las propuestas y resultados de la bibliografía que hay discrepancias entre las clases que determinan los distintos criterios. 
En el presente trabajo se parte de los resultados del análisis y clasificación de un corpus de esquemas argumentales correspondientes a construcciones agentivas, en el que se han determinado numerosas clases de carácter síntactico-semántico, muchas de las cuales se caracterizan por la presencia de un CD afectado. De la observación de estos resultados se han obtenido datos sobre tipología, combinatoria y alternancias en los esquemas argumentales que a veces contrastan con los datos de la bibliografía sobre argumentos afectados, datos a partir de los cuales se ofrece aquí un breve análisis.

\section{CRITERIOS DE ANÁLISIS DEL CORPUS}

El corpus está compuesto por un total de 1914 esquemas con estructura argumental mínima, establecida mediante diversas pruebas de extracción (Cuartero, en prensa). Los esquemas están formalizados por medio de una serie de variables (alguien / algo / algún lugar / algún tiempo / algún modo etc.) (Devís, 2001 y Báez, 2002) y clasificados a partir de los datos de selección sintáctica y semántica de los verbos, de las posibilidades de alternancia de sus estructuras y de su subclase aspectual (Cuartero, 2003 y en prensa). Para la caracterización de esquemas con CD es importante distinguir tres fenómenos:

1. Algunos presentan alternancia recesiva (= anticausativa o ergativa): el verbo da lugar a una estructura transitiva con un argumento causa y otro paciente (alguien hierve algo; alguien hunde algo) y a otra intransitiva y sin argumento causa (algo hierve - algo se hunde).

2. Algunos están asociados a perífrasis resultativas, es decir, a construcciones con estar + participio o estar + adjetivo (alguien hunde algo - algo está hundido; alguien limpia algo - algo está limpio), que presentan tres posibilidades de relación temporal con el evento correspondiente (Cuartero 2003):

- perífrasis perfectiva, si el resultado es posterior a la realización del evento:

alguien ha roto algo - algo está roto,

- perífrasis imperfectiva, cuyo resultado es sólo simultáneo a la realización del evento:

alguien entretiene a alguien - alguien está entretenido,

- perífrasis perfectiva-imperfectiva, cuando el resultado se da tanto simultánea como posteriormente a la realización del evento:

alguien asusta / ha asustado a alguien - alguien está asustado.

3. Finalmente, se dan cuatro tipos aspectuales de esquemas observables por su compatibilidad con los operadores en $x$ tiempo y durante $x$ tiempo:

- esquemas gnómicos, incompatibles con ambos: 
alguien tiene algo *en $x$ tiempo /*durante $x$ tiempo

- esquemas télicos, compatibles sólo con en $x$ tiempo:

alguien alcanza algo en $x$ tiempo / *durante $x$ tiempo

- esquemas atélicos, compatibles sólo con durante $x$ tiempo:

alguien mira algo / a alguien *en $x$ tiempo / durante $x$ tiempo

- esquemas télico-atélicos, compatibles con ambos:

alguien le habla a alguien de algo en x tiempo /durante x tiempo'.

\section{CRITERIOS DE DETERMINACIÓN DE LOS OBJETOS AFECTADOS}

Desde un punto de partida funcionalista, para el análisis del corpus de esquemas agentivos se aplicó la prueba ¿Qué le hace X a Y? (Fillmore, 1968: 4), complementada por una más general ¿Qué le sucede a $Y$ ? (Chafe, 1970: 160): ambas determinan la clase de los esquemas con argumentos afectados ${ }^{2}$. En el corpus se localizan, de un total de 1914 esquemas, 1001 con CD afectado, 546 con CD no afectado y 72 con $C D$ efectuado. En cuanto a las características de estas tres categorías se observan algunas restricciones y generalidades:

- Los argumentos afectados nurica pueden ser realizados por cláusulas sustantivas. Se aplica una generalización: si un esquema puede aparecer determinado por un $\mathrm{CD}$ clausal, se concluye automáticamente el carácter no afectado del complemento ${ }^{3}$.

- Los argumentos efectuados no son afectados. Si bien las dos clases tienen propiedades comunes (se asocian a argumentos causa y a alternancias anticausativas), los efectuados no cumplen las dos pruebas y, dependiendo del carácter léxico del verbo, pueden ser realizados por cláusulas (la confusión produjo que no pudieran encontrarse).

' Se han observado, además, correspondencias cntre los tipos de resultados y los tipos aspectuales (Cuartero, 2003): los esquemas gnómicos nunca tienen perífrasis resultativa; los télicos con perífrasis, sólo la tienen posterior al evento; la perílrasis de los atélicos sólo puede ser simultánea y los télicoatćlicos con perífrasis, pueden tenerla de cualquier tipo.

${ }^{2}$ Los argumentos afectados pueden realizar las funciones sintácticas sujeto, complemento directo, complemento indirecto o complemento preposicional.

${ }^{3}$ Asimismo la mayor parte de los esquemas con CD no afectado, siempre que éste no indique una cantidad (pesa quince kilos) o el lugar en relación al que se produce un movimiento (recorrimos el pais) presentan la posibilidad de realizarlo como cláusula en los enunciados (p. ej. verbos de percepción, de comunicación etc.). 


\section{TIPOS LÉXICOS DE ESTRUCTURAS CON OBJETOS AFECTADOS}

Los intentos de descripción por extensión de los objetos afectados remiten de un modo u otro a la formulación de Anderson (1977: 15): "Affected is used in an extended sense to mean changed, moved, altered in status or created". Ya que la Gramática de Casos distingue entre tema (paciente o afectado) y factitivo, en algunos trabajos se producen discrepancias en cuanto a si se consideran los argumentos afectados como una categoría que incluye a pacientes y a factitivos o bien los pacientes constituyen una categoría que engloba afectados y factitivos o bien se separan, como en este trabajo, los afectados (= pacientes) de los efectuados. Se observa, con diferentes enfoques, en formulaciones como las de Jaeggli (1986: 608), Roberts (1987: 210), Fellmann y Zribi-Hertz (1989: 28), Tenny (1994: 158) o, entre autores que se ocupan del español, Demonte (1991: 37-38) y Campos (1999: 1532).

En el modelo que presenta Langacker (1991: 285), muestra un esquema canónico de un evento transitivo como un cambio de propiedades en un argumento (afectación) que está causalmente producido por otro argumento.

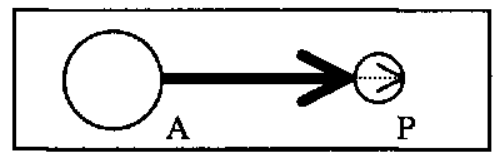

Al comparar los resultados del análisis del corpus con las descripciones al uso de las categorías de objetos afectados, se observa que hay más clases de las hasta aquí enumeradas. La clasificación resultante implica en principio ocho tipos más uno, cuyas propiedades se presentan en la siguiente tabla y se desarrollan a continuación ${ }^{4}$.

\begin{tabular}{|c|c|c|c|c|c|}
\hline clase $^{5}$ & recesivo & télico & atélico & $\begin{array}{c}\text { resultado } \\
\text { imperfectívo }\end{array}$ & más de dos argumentos \\
\hline 1. destruido & \multirow{5}{*}{ Sí } & \multirow{6}{*}{ Sí } & NUNCA & NUNCA & \\
\hline 2. transformado & & & \multirow{5}{*}{ POSIBLE } & \multirow{4}{*}{ POSIBLE } & NO \\
\hline $\begin{array}{l}\text { 3. cambiado de } \\
\text { posición }\end{array}$ & & & & & POSIBLE \\
\hline 4. desplazado & & & & & $\begin{array}{c}\text { SIEMPRE } \\
\text { (LOCATIVOS) }\end{array}$ \\
\hline 5. unido / separado & & & & & SIEMPRE \\
\hline 6. transferido & \multirow{3}{*}{ NUNCA } & & & NUNCA & (AFECTADOS) \\
\hline 7. contactado & & \multirow{2}{*}{ NO } & \multirow{2}{*}{ Sí } & \multirow{2}{*}{ SIEMPRE } & \multirow{2}{*}{ NO } \\
\hline 8. en relación & & & & & \\
\hline
\end{tabular}

\begin{tabular}{|l|l|l|l|l|l|}
\hline ix. mantenido & Sí & NUNCA & sí & SIEMPRE & Sí \\
\hline
\end{tabular}

^El modelo se ha extraído del corpus de verbos agentivos, pero parece igualmente aplicable a los procesos.

${ }^{5}$ Las propiedades se indican en la tabla de manera escalar: siempre (característico), sí (típico), posible (no marcado), no (típico) y nunca (característico). 
Los más característicos, en vista del tratamiento que han recibido, son los transformados, prototípicamente formalizados en esquemas biargumentales, y los desplazados, siempre en esquemas con más argumentos; no obstante, hay otros tipos de esquemas típicamente biactanciales afectadores, pero que no indican cambios de propiedades $(7-8)$ y finalmente objetos mantenidos en su estado o localización (ix). Asimismo, unas clases muestran bastantes propiedades privativas (siempre / nunca) y otras ninguna. Los objetos están más claramente afectados en las posiciones de más arriba de una escala gradual que presenta relaciones de encadenamiento, ya que por la parte de abajo, la clase de objetos en relación con otro(s) empieza a mostrar características comunes con esquemas no afectadores. A veces sólo aparecen límites difusos entre las categorías (con verbos en posiciones intermedias), mientras que otras veces presentan límites claros (entre siempre / nunca y las demás marcas).

\section{ESTRUCTURAS ARGUMENTALES CON CD AFECTADO}

Como se observa en la tabla anterior, los tipos de más arriba (1-2) y más abajo (7-8) se relacionan prototípicamente con estructuras biargumentales, mientras que los demás (3-6) se caracterizan por la presencia de más de dos argumentos. Ello es específico de los verbos que implican un cambio de situación del objeto en un sentido amplio, los de las clases 3-6. Cambiado de posición es una categoría con características intermedias entre 2 y 4, ya que no necesariamente implica un argumento de referencia local (enrollar algo, doblar algo); si lo hace, éste puede ser direccional (girar / inclinar algo hacia algo / algún lugar) o locativo (tumbar algo en algún lugar). Indica un movimiento intrínseco, frente a la clase desplazado, que indica un desplazamiento, movimiento extrínseco en coordenadas espaciales vectoriales orígen, vía, dirección y meta (alguien lleva algo de algún lugar por algún lugar a / hacia algún lugar). Se produce un tránsito hacia la clase unido / separado donde también hay desplazamiento pero con referencia a otras entidades y no a localizaciones espaciales (alguien une algo a algo / con algo). El límite aquí es muy vago: un mismo verbo puede tener esquemas argumentales de este tipo y del anterior (alguien aleja algo y algo; alguien aleja algo de algún lugar). El tipo transferido implica siempre un tercer argumento, ya que hay un cambio de poseedor del CD que se da entre el sujeto y un tercer argumento.

La posición en la tabla de la clase conservado es muy específica: se contrapone a todas las que producen un cambio en el objeto $(1-6)$, puesto que implica la presencia de otro argumento locativo o una especificación de tipo atributivo sobre el estado del argumento afectado. 


\section{FENÓMENOS COMBINATORIOS RELACIONADOS CON LA AFECTACIÓN DE LOS CD}

Se ha comprobado en distintos trabajos que la presencia de una entidad afectada como objeto (argumento interno) en la estructura argumental de un verbo tiene influencia en las propiedades de combinación de los elementos. Se nombran varios fenómenos: la telicidad característica de las construcciones con objeto afectado (en Tenny, 1987 y 1994), la posibilidad exclusiva de dar lugar a estructuras anticausativas para verbos con argumentos afectados, las construcciones con sustantivos deverbales (nominalizaciones) y las construcciones con participios (adjectival passives) (en Doron y Rappaport-Hovav, 1991).

6.1. En dos trabajos de Tenny (1987 y 1994) se insiste en que lo característico de los objetos afectados es su función de delimitador temporal del evento, por lo que todos se asocian a la telicidad del evento (1994: 158): "An affected argument [...] makes the event described by the verb delimited, by undergoing a change of state that marks the temporal end of the event. [...] It is clear that affected arguments are measuring arguments. [...] An affected argument is one that measures out and imposes delimitedness on the event".

Como se ve en la tabla de arriba, eso sólo se cumple con unos tipos específicos de afectados, y nunca con los tipos $7 \rightarrow 8$, que están en esquemas y enunciados atélicos. Además, no se puede aceptar que cualquier CD de un enunciado télico se deba considerar afectado (leyó el libro en dos horas). Asimismo, un evento que implica un cambio de carácter reversible (de propiedad o locación) en el argumento afectado determina su adscripción a la clase de los télico-atélicos (calentó la sopa en un minuto / durante un minuto; saqué al niño al balcón en un minuto / durante un minuto). Finalmente, la aspectualidad de los enunciados con verbos que indican movimiento extrínseco depende tanto de los complementos locales de cada enunciado (lo llevó a casa en una hora /*durante una hora; lo llevó hacia casa ${ }^{*}$ en una hora / durante una hora) como del carácter del verbo (lo pasea por el parque en una hora / durante una hora). Los tipos contactado (alguien acaricia a alguien) y mantenido (alguien mantiene algo en un lugar), por ejemplo, sufren un tipo de afectación que sólo dura lo que la realización del evento, por lo que se trata de esquemas típicamente atélicos.

6.2. Los verbos con objeto no afectado no entran en construcciones recesivas (= ergativas $o$ anticausativas) ni en las llamadas pasivas nominales debido a las restricciones por afectación (affectedness constraint) (Jaeggli, 1986: 607): "If a complement of $\mathrm{X}$ is unaffected, it is impossible to eliminate the external $\theta$-role of $\mathrm{X}^{\prime \prime}$. Esta restricción alcanza a los procedimientos de anticausativización ${ }^{7}$, si bien

${ }^{6}$ En la misma linea, Doron y Rappaport-Hovav (1991: 83) proponen la separación de argumentos en la estructura eventiva: " $y$ is an affected argument of $V(x, y)$ if the event-structure of $V$ contains a sub-eventuality e such that $y$, but not $x$, is an argument in $e^{\text {". }}$ 
algunos trabajos se han ocupado de defender la idea de que estas restricciones no son válidas para las lenguas románicas (Zubizarreta, 1987).

El problema en este caso viene dado por la confusión entre muy diversos tipos de construcciones: las pasivas reflejas y las impersonales con se por un lado, que no son inacusativas, sino medias (vid. Fagan, 1988 y Demonte, 1991: 36-63) y en las que el argumento externo (el sujeto causativo) en realidad no se elimina, frente a las construcciones recesivas, las verdaderas anticausativas, en las que no se puede considerar la presencia de un argumento causa. En palabras de Mendikoetxea (1999: 1586): "Existen [...] diferencias fundamentales de significación entre las oraciones pasivas y las oraciones inacusativas [anticausativas] en cuanto al grado de presencia / ausencia del sujeto nocional implícito". Por supuesto, un problema añadido en español es la ambigüedad de las formas, pero la distinción se subjetiva con tests de compatibilidad de la construcción o bien con elementos como él solo / por sí sólo o bien con modificadores agentivos (que indican la participación voluntaria del argumento agentivo en la realización del evento, como voluntariamente, intencionadamente etc.) y con cláusulas finales (si bien los dos últimos sólo son válidos para esquemas agentivos):

El barco se hundió él solo / por sí solo (anticausativa, no hay agente)

El barco se hundió intencionadamente (pasiva, hay un agente oculto)

El barco se hundió para cobrar el seguro (pasiva, hay un agente oculto).

No pueden ser inacusativas o recesivas las construcciones que "no pueden expresar eventos que se realizan de manera espontánea sin la intervención volitiva de un agente. Oraciones como Se construyó un puente y Se divulgaron los numores sólo pueden tener una interpretación pasiva con se y no una interpretación inacusativa [recesiva] incoativa" (Mendikoetxea, 1999: 1587). Por los resultados del análisis del corpus parece claro que la alternancia causativa es una propiedad privativa que se adscribe sólo a eventos en cuyo esquema aparece un argumento afectado con la función CD, sin embargo, no todos los esquemas con CD afectado presentan, por diversas razones, esta alternancia que, como se muestra en la tabla anterior, nunca se da con las clases $6-8$, mientras que resulta típica de las restantes.

6.3. Por lo que respecta a las construcciones con sustantivos deverbales, resulta claro que en español no es aplicable la restricción por afectación, si se consultan simplemente los ejemplos de nominalizaciones con los que trabajó Demonte (1991: 45-46):

- en un caso corresponden a los eventos intransitivos con sujeto afectado (el aburrimiento de Maria: i. e. María se aburrió; el horror de los viajeros: i.e. los viajeros se horrorizaron) pero precisamente no a los transitivos con CD afectado (*el aburrimiento de María por la película: $i$. e. la película aburrió a María; *el horror de los viajeros por la tormenta: i.e. la tormenta horrorizó a los viajeros);

7 "Anticausativization deletes the agentive external argument of a transitive verb" (Zubizarreta, 1985). 
- en otro caso corresponden a verbos transitivos con CD no afectado que sólo en algunos casos permiten nominalizaciones (la visión del volcán me emocionó; *la mirada del volcán me horrorizó) y cuyas excepciones parecen tener más relación con los elementos léxicos que con la semántica del verbo.

6.4. En cuanto a las construcciones con participios, que citan Doron y Rappaport-Hovav (1991:91) como fenómeno dependiente de la presencia de argumentos afectados, en español no hay pruebas que permitan defender esa posibilidad: se dan construcciones de participios tanto con argumentos afectados (un coche roto) como con no afectados (un libro leido; un chiste muy repetido, un problema previsto etc.). Los propios ejemplos de las dos autoras muestran la poca generalidad de esa hipótesis.

\section{BIBLIOGRAFÍA}

Anderson, M. (1977), NP Pre-Posing in Noun Phrases. NELS, 8, 12-21.

Báez, V. (2002), Desde el hablar a la lengua: Prolegómenos a una teoría de la sintaxis y la semóntica textual y oracional. Málaga: Ágora.

Campos, H. (1999), Transitividad e intransitividad, en I. Bosque y V. Demonte (eds.), Gramática descriptiva de la Lengua Española. Madrid: Espasa-Calpe, 1519-1574.

Chafe, W. L. (1970), Meaning and the structure of language. Chicago: Chicago University Press.

Cuartero, J. (2000), Algunas consideraciones sobre la agentividad en español. Anuario de Estudios Filológicos, XXIII, 65-76.

- (2003), Clases aspectuales y perifrasis resultativas en español, en M. Emsel y A. Hellfayer (eds.), Brückenschlag. Gerd Wotjak zum 60. Geburtstag. Frankfurt: Lang, 55-62.

- (en prensa), Cosas que se hacen: Esquemas sintáctico-semánticos agentivos en español. Frankfurt: Lang.

Demonte, V. (1991), Detrás de la palabra. Madrid: Alianza.

Devís, P. P. (2001), Fundamentos teóricos básicos de morfología y semántica oracionales. Málaga: Ágora.

Doron, E. y M. Rappaport-Hovav (1991), Affectedness and Externalization. NELS, 21, 81-94.

Fagan, S. (1988), The English Middle. Linguistic Inquiry, 19, 181-203.

Fell baum, Ch. y A. Zribi-Hertz (1989), La construction moyenne en francais et en anglais: étude de syntaxe et de sémantique comparées. Recherches Linguistiques à Vincennes, 18, 19-57

Fillmorc, Ch. (1968), The case for case, en E. Bach y R. T. Harms (eds.), Universals in linguistic theory. London-New York: Holt, Rinehart \& Winston, 1-88.

Jaeggli, O. (1986), Passive. Linguistic Inquiry, 17, 587-622.

Langacker, R. L. (1991), Foundations of Cognitive Grammar: Descriptive Application. Stanford: Stanford University Press.

Mendikoetxea, A. (1999), Construcciones inacusativas y pasivas, en I. Bosque y V. Demonte (eds.) Gramática descriptiva de la Lengua Española. Madrid: Espasa-Calpe, 1575-1630.

Roberts, I. (1987), The Representation of Implicit and Dethematized Subjects. Dordrecht: Foris.

Ten ny, C. L. (1987), Grammaticalizing Aspect and Affectedness. Cambridge: MIT.

- (1994), Aspectual Roles and the Syntax-Semantics Interface. Dordrecht: Kluwer Academic Publishers.

Zubizarreta, M. L. (1985), The Relation between Morphophonology and Morphosyntax: The Case of the Romance Causatives. Lingustic Inquiry, 16, 247-289.

Zubizarreta, M. L. (1987), Levels of representation in the lexicon and in syntax. Dordrecht: Foris. 